\title{
Subject Index Vol. 25, 1996
}

Acanthosis nigricans 252 Achondroplasia 221 Acute subdural hematoma 123 Anterior sacral meningocele 143 Apert syndrome 20 Arachnoid cysts 165 Arteriovenous fistula 252 Artifact 156 Astrocytoma 214 Atretic cephalocele 260 Autologous bone marrow transplantation 7

Bone plates, complications 31 Brain 100

development 130

neoplasms 64

stem auditory evoked potentials 227

- glioma 45

- infection 8

- invasion 182

- tumor(s) 83,210

tumor 109

Carotid artery 188 Cavernous angioma 137

- malformation 151

Cell adhesion kinase 64

Central nervous system 100

Cephalocele 260

Cerebral arteriovenous malformation 277

hemorrhage 277

palsy 28,233 Cerebrospinal fluid 73 Cervical spine 20 Chemotherapy 174

-, high-dose 7

Chiari malform
$-\quad$ I 252

ation(s) 221

II 221 Chick embryo 130

abuse 116 Childhood 240

Chronic subdural hematoma 137 Complications, tumor surgery 41 Compute(rize)d tomography 94

256 Congenital malformation 36

spinal fusion 20 Cranial meningocele 260

window model 123 Craniofacial syndrome 78 Craniopharyngioma 265

Craniosynostosis 20, 252 Craniotomy 31, 78 Currarinno's triad 143 Cystic optic chiasm fibrillary astrocytoma 265 Cystoperitoneal shunt 165

Dandy-Walker malformation 260 Diffuse cerebrospinal gliomatosis

94 Dorsal rhizotomy 233 Dural cavernous angiomas 105 Duraplasty 221

Electrophysịjology 233 Encephalocele 130, 260 Endoscopy 57 Ependymoma 7,210 Epidemiology 240 Epidural hematoma 302 Ewinng's sarcoma 100 Extent of resection 182 Extracerebral cavernous angiomas 105 Extraperitoneal CSF collection 160

Familial cephalocele 260

- malformation

Fenestration 165

Fibroblast growth factor receptor

252 Fracture 256

Genetic counseling 269 Gliomatosis cerebrị 94 Growth factor receptors 64

Halo 256

Head injury 116,302,309

- trauma 156

Hemorrhage 151

Histopathology 123

Hydrocephalus $57,73,165,248$

Immunocytochemistry 137

Incidence 240

Infant 116

Injury 188

Intestinal obstruction 160

Intracranial cavernous angioma

105 Intraoperativeelectrophysiologic

monitoring 13

- ultrasound 221

Intrinsic brain stem tumors, surgery 41

Jugular stenosis 252

Lambdoid synostosis 1

Magnetic resonance imaging 45, 94, 151,286

--, transsphenoidal menin-

goencephalocele 54 Medulloblastoma 174,182,210 Medullomyoblastoma 214 Meninges 100

Meningoencephalocele, transsphenoidal type 54 Meningomyelocele, infants 227 Myelomeningocele 295

Neural tube defects 260 Neuroendoscopy 248 Neuroradijology 302 
Occipital plagiocephaly 1 Occult vascular malformations 83 Odontoid fracture 256

process 2560 perative position 210

technique, craniotomy 31 Outcome prediction, medulla tumor

surgery 41

Pediatric brain neoplasms 64

- tumors 45

cavernous angiomas 105

scoliosis 295

stroke 277

tumors 182 Pediatrics 57 Piglet 123

Pilocytic astrocytoma 109 Polymerase chain reaction 64 Posterior fossa 105

- decompression 221

- tumor 210 Postoperative deficits, intrinsic

brain stem tumors 41 Prenatal hydrocephalus 36 Primary intracranial tumor 240 Primitive neuroectodermal tumor(s)

CNS 174

Prone position 286

Radiation 214

- therapy 174

Radiotherapy 109

作 78 Recurrent hemorrhage 137 Relapse 7

Retinal hemorrhage 116 Retinoblastomas 269 Rhizotomy 13

Sacral anomaly 143 Sagittal synostosis 78 Selective dorsal rhizotomy 28 Sellaturcica 265 Sellarmass 269 Sequential CT scan 94 Shunt complications 160 malfunction 315

pumping test 73 Skull X-ray 156 Skull/cranium 100 Spasticilty 13,233 Spinabifida 57,286 Spinal cord 214

involvement 94

stenosis 28 Spine 100

deformity 295 Spleen 36

Stereotactic biopsy 94 Stroke 188

Subdural hematoma 116 Surgery 214 Synchondrosis 256

Tethered cord 286

- syndron 295 tivator 137 Transbalal approach, meningoencephalocele 54 Trauma systems 309 Triage 309

Trilateral retinoblastoma 269 Tyrosine kinases 64

Unilateral hydrocephalus 248 Urokinase 315

Valve 73

Vascular abnormalities 147

endothelium 137

malformations 147 Ventriculoatrial shunt 315 Ventriculoperitoneal shunt 315 Vertebral artery 147

fusion 20

\section{KAIIVGEÍl}

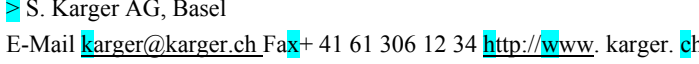

